\title{
Characterisation of a Composite LEPS
}

\author{
Moumita Roy Basu ${ }^{a}$, Sudatta Ray ${ }^{b *}$, Abhijit Bisoi $^{b \dagger}$, M. Saha Sarkar ${ }^{b \ddagger}$ \\ ${ }^{a}$ University of Calcutta, \\ Kolkata - 700073, INDIA \\ ${ }^{b}$ Saha Institute of Nuclear Physics, \\ Bidhannagar, Kolkata - 700064, INDIA \\ E-mail: maitrayee.sahasarkaresaha.ac.in
}

\begin{abstract}
A low energy photon spectrometer (LEPS), which is a composite planar HPGe, has been characterised experimentally. It has been shown that beyond $200 \mathrm{keV}$, effect of image charges deteriorates the efficiency of the detector in its addback mode. Data have been corrected on eventby-event basis resulting in improvement of the performance.
\end{abstract}

KEYWORDS: : Gamma detectors (scintillators, CZT, HPG, HgI etc); X-ray detectors; Interaction of radiation with matter; Data processing methods.

${ }^{*}$ Presently at Amity University, Noida - 201303, INDIA

${ }^{\dagger}$ Presently at Indian Institute of Engineering Science and Technology, Howrah - 711103, INDIA

\#Corresponding author. 


\section{Contents}

1. Introduction 1

2. Experimental Details 1

2.1 The detector 1

2.2 Modes of detection in a composite detector

2.3 The Experiment and Data Analysis

3. Results and Discussion 5

3.1 Relative efficiency 5

3.1.1 Causes

3.1.2 Image charge formation

3.1.3 Correction 7

3.1.4 Addback Factor 8

4. Conclusion 8

\section{Introduction}

Apart from a few medium sized array [1], gamma detector arrays usually have very low detection efficiency for photons with energies less than $50 \mathrm{keV}$ due to the absence of suitable detectors which are efficient at these low energies. However, this energy range has special importance for detection of (i) X-rays for identification of fission- fragments, (ii) highly converted low energy gammas emitted from isomers in medium and heavy nuclei, (iii) gamma transitions connecting the lowest states in heavy nuclei, etc. The low energy photon spectrometers (LEPS) having reasonable resolution at this energy range have very low efficiency. So it is desirable to use a composite detector which includes more than one planar LEPS having good resolution to get higher efficiency. Electrically segmented planar LEPS has been used in some of the arrays as a solution to this problem. In this paper, a segmented planar LEPS will be characterized.

\section{Experimental Details}

\subsection{The detector}

In the present work; a composite planar HPGe low energy photon detector (designated as "Composite" along the text) [3] with four segments mounted on a common cryostat (Table 1) similarly toa Clover detector has been used. The detector has been fabricated by DSG Detector Systems 

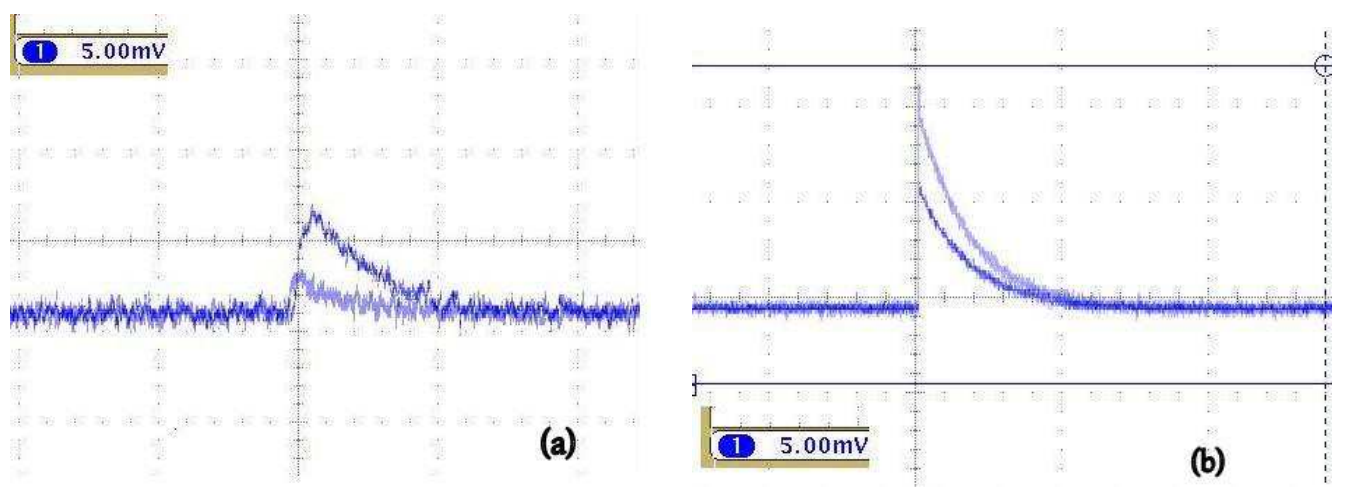

Figure 1. Preamplifier outputs of one of the segments of the Composite detector, (a) without and (b) with bias.

Company ${ }^{1}$, Germany. The crystal in this planar detector is of p-type. The volume of each crystal (segment) is such that each of them has a resolution of around $500 \mathrm{eV}$ for $122 \mathrm{keV}$. The efficiency of the total system is around 4 times that of a single detector. The active area is $4 \times 80 \mathrm{~mm}^{2}$. It has a thin Be window (Table 1), to maximize the low energy efficiency of the detector. The necessary reverse bias for this composite LEPS for operation is very low, only $-300 \mathrm{~V}$. Bias always depends on the material, crystal quality and design. This low bias for optimum performance indicates that the crystal quality is good. The Composite detector use a single bias supply. The detector assembly is designed such that the supply provides reverse voltage to four segments.

The noise level is quite low even without bias. Fig. 11 shows the preamplifier output from one of the crystals without bias as well as the same preamplifier output with $-300 \mathrm{~V}$ detector bias.

In this work the performance of this detector with a normal planar HPGe detector (ORTEC GLP 10180/07 SH GLP: designated as "Old LEPS") will be compared. Specifications of these two detectors are shown in Table 1 .

\subsection{Modes of detection in a composite detector}

The total full energy peak efficiency of any composite detector with more than one crystal (segment) includes the effect of two complimentary processes [2]. They are

- single fold events, when full gamma energy is deposited in any one of the individual crystals. This is related to the direct full energy peak detection efficiencies of individual crystals,

- the full gamma energy resulting from the partial absorption in two or more crystals through Compton effect (and/or pair production process followed by escape of one/both of the 511 $\mathrm{keV}$ gamma rays for high energy gammas). This is related to the coincidence detection efficiency.

Direct detection efficiency of a composite detector is obtained by treating the signals from individual crystals separately. It is obtained from the uncorrelated sum of spectra from each of

\footnotetext{
${ }^{1}$ DSG Detector Systems GmbH, Robert Bosch Strasse 38, 55129 Mainz, Telephone: 061315075 30, Fax: 06131 5075 40, email: info [at] detectorsystems.de. The company has pulled back from operations.
} 


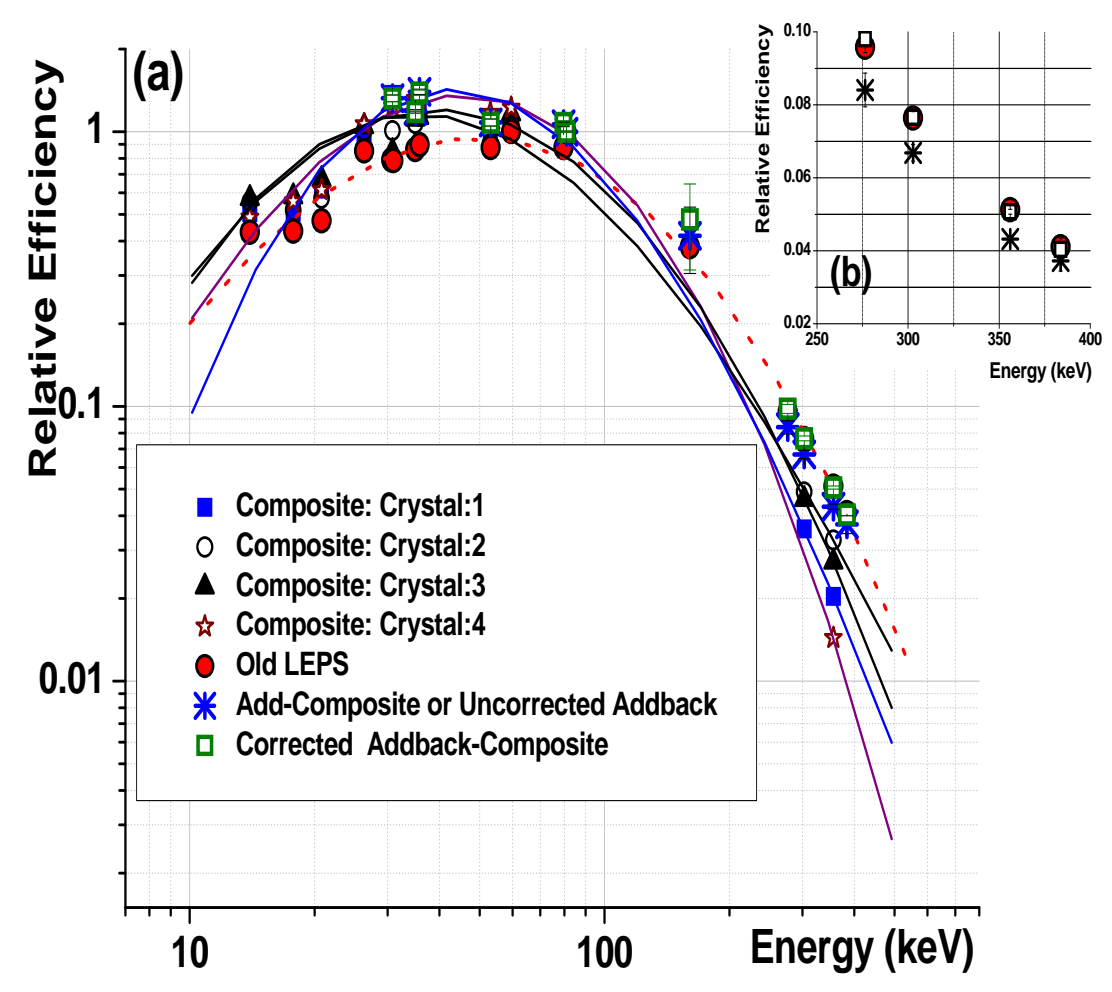

Figure 2. (a) Comparison of relative efficiencies of individual crystals of Composite LEPS in singles, add, addback and corrected addback modes with that of the Old LEPS. (b) (Inset) The differences between relative efficiencies of Old LEPS and Composite LEPS, in add and corrected addback modes have been displayed in an expanded scale for clarity. The efficiencies have been normalised to 1 at $81 \mathrm{keV}$.

these crystals. On the other hand, in coincidence detection mode, two or more crystals are in temporal coincidence, mainly due to Compton scattering from one crystal to its neighbouring ones. The energy signals of individual crystals fired simultaneously, are recorded event by event in the list mode. The coincidence detection efficiency is determined from the spectra (addback spectra) generated from time correlated sum (addback) of these signals. So the total full energy peak detection efficiency of this composite detector is $\varepsilon_{\text {total }}=\varepsilon_{\text {direct }}+\varepsilon_{\text {addbk }}$. Therefore, at relatively higher energy, where Compton processes become important, the addback contribution is responsible for the enhancement of the total full energy peak detection efficiency compared to the direct mode. As a measure of this enhancement, the addback factor $\mathrm{F}$ is defined as the ratio of the total full energy peak efficiency to the direct detection efficiency; i.e., $\mathrm{F}=\varepsilon_{\text {total }} / \varepsilon_{\text {direct }}$.

\subsection{The Experiment and Data Analysis}

We have used ${ }^{152} \mathrm{Eu},{ }^{133} \mathrm{Ba}$ and ${ }^{241} \mathrm{Am}$ radioactive sources to determine the relative efficiency of the detectors. The efficiency of the composite detector has been determined from the sum spectrum generated by addition of independent singles spectra of four segments. We have designated this sum spectrum as $A d d$ spectrum and this mode as $A d d$ mode. In Clover detectors, at around $1 \mathrm{MeV}$, the addback improves the absolute efficiency by about 1.5 times [2]. It means that the efficiency 
Table 1. Specifications of the two detectors used in the present work

\begin{tabular}{|c|c|c|c|c|c|c|c|}
\hline \multicolumn{3}{|c|}{ Detector } & \multicolumn{3}{|c|}{ Dimensions } & \multicolumn{2}{|c|}{ Be-Window } \\
\hline Make & Type & $\begin{array}{c}\text { High } \\
\text { Voltage }(\mathrm{V})\end{array}$ & $\begin{array}{l}\text { Dia. } \\
(\mathrm{mm})\end{array}$ & $\begin{array}{c}\text { Height } \\
(\mathrm{mm})\end{array}$ & $\begin{array}{c}\text { Area } \\
\left(\mathrm{mm}^{2}\right)\end{array}$ & $\begin{array}{l}\text { Dist. }^{2} \\
(\mathrm{~mm})\end{array}$ & $\begin{array}{l}\text { Thick } \\
(\mathrm{mm})\end{array}$ \\
\hline ORTEC & $\begin{array}{c}\text { GLP } \\
\text { Planar: n-type } \\
\text { single }\end{array}$ & -1000 & 10 & 7 & 80 & 5 & 0.127 \\
\hline DSG & $\begin{array}{l}\text { PGP 4seg80-7 } \\
\text { Planar: p-type } \\
4 \text { segments }\end{array}$ & -300 & 24 & 7 & $4 \times 80$ & 5 & 0.127 \\
\hline
\end{tabular}

${ }^{2}$ Distance between the crystal and the endcap

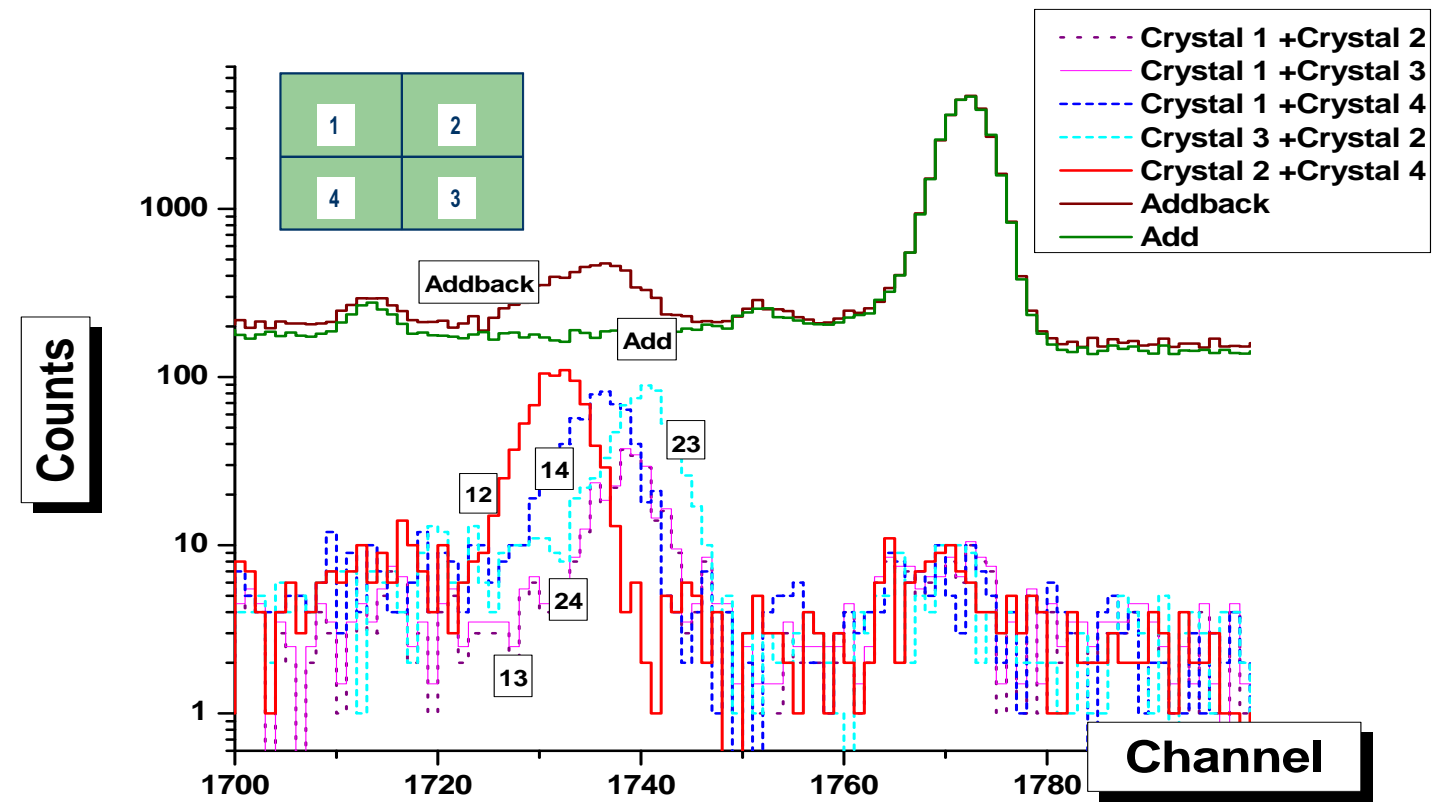

Figure 3. The comparison of add, addback and different two-fold spectra $(12,14,23,24$ and 13) for the Composite LEPS. The two -fold spectra 12 (say,) is generated from simultaneous events in segments 1 and 2. The segments numbers are indicated in the inset figure.

in the Addback mode is 1.5 times more than that in the Add mode. However, this ratio is energy dependent. At low energies, where Compton effect is not dominant, addback process is not advantageous. To take advantage of the addback mode (as discussed earlier in Section 2.2) at energies above $200 \mathrm{keV}$ where the efficiency of a small area planar detector falls sharply, correlated total spectrum of the four crystals (similar to addback spectrum of a Clover) has been generated. In this case, the Addback spectrum is generated by event wise summing of correlated data obtained in four 
segments of the composite detector. We have designated this mode as Addback (uncorrected) in the figures and text. We have collected the data from the composite detector in the LIST Mode. In this work, the relative efficiency of the composite detector in Add and correlated Addback modes have been compared with the relative efficiencies of individual crystals and that of Old LEPS)(Fig.Дa).

\section{Results and Discussion}

\subsection{Relative efficiency}

It has been found that any single crystal of the composite LEPS (Fig. 27a) or the summed spectra of the four crystals show almost similar relative efficiencies at low energies $(\leq 100 \mathrm{keV})$ when compared with the Old LEPS. It is observed that for the composite detector, the relative efficiency in the Addback is exactly similar to that in the $A d d$ mode. So they are indicated as Add-Composite (uncorrected Addback) in Fig. 2. No improvement is noted beyond $200 \mathrm{keV}$. It is even worse than the Old LEPS (Fig. 2 b). At higher energies the efficiency of the Old LEPS improves and is better than the Add (Addback-uncorrected) modes of the composite detector. This is an unexpected observation and needs closer inspection to determine its cause.



Figure 4. The single- and two-fold spectra are compared for a ${ }^{133} \mathrm{Ba}$ source.

\subsubsection{Causes}

The add, addback, single- fold and two - fold spectra (Figs. 3, 4) have been compared to understand this feature. It is observed that the addback spectrum of the composite LEPS contains a relatively low intensity shadow peak corresponding to each higher energy gamma peak (Fig. 3). While comparing the spectra generated from single- fold and two- fold events only, it is observed that 
these peaks are solely generated by the two fold events as evident from Fig. 4 . The two -fold spectrum (Fig.4) does not contain the photopeak, it only includes the shadow peak corresponding to it.

To identify the origin of these shadow peaks more accurately, two- fold spectra generated from neighbouring crystals $(12,14$ and 23$)$ as well as diagonally opposite crystals $(13,24)$ have been compared in Fig.3. It is clearly seen the neighbouring crystals are contributing nearly double of that contributed by the diagonally opposite ones in the shadow peak. The peak-shifts vary as a function of second crystal position with respect to that of the first crystal depending on the position of the source.

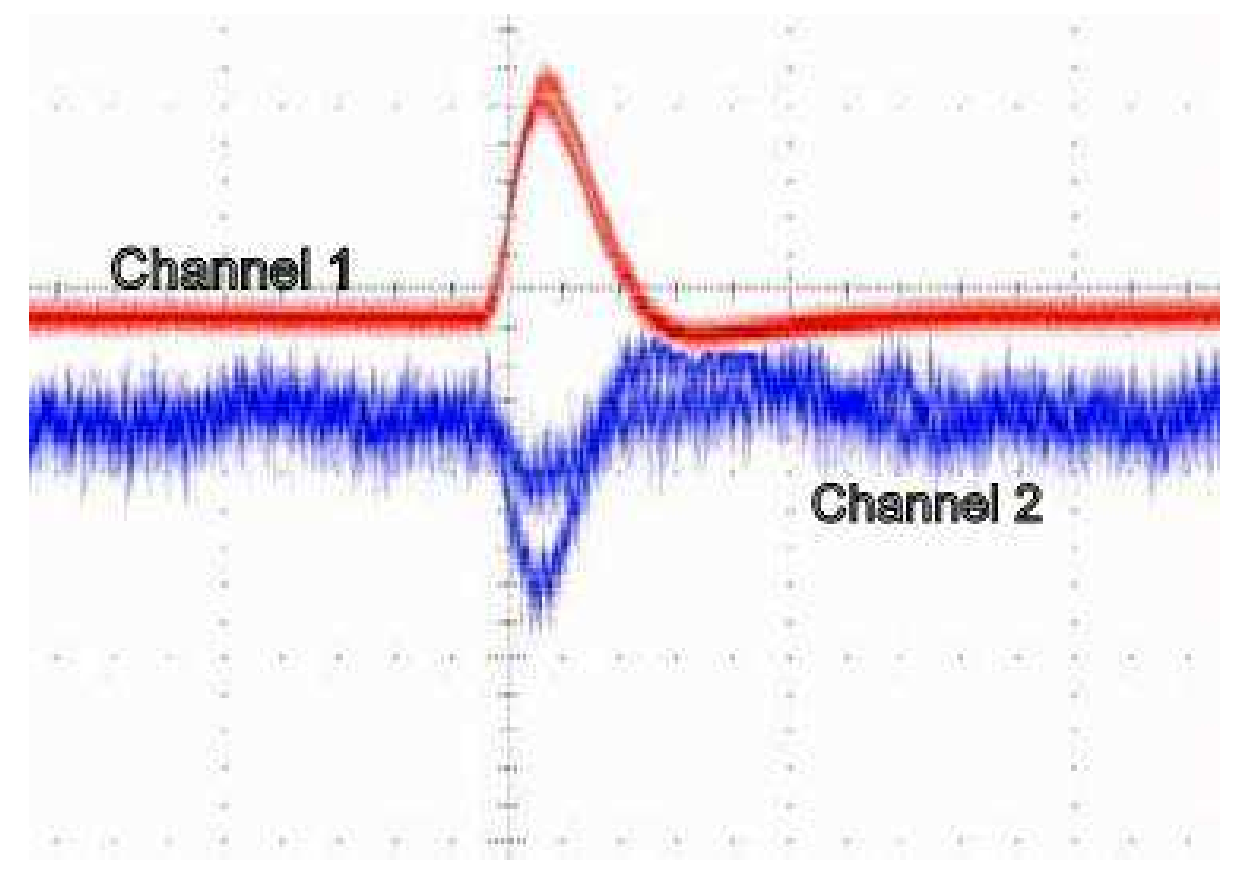

Figure 5. Amplifier (negative) outputs in Channel 2 corresponding to Image pulses (negative) generated in the neighbouring segment (say, 2) are observed simultaneously if positive amplifier outputs corresponding to the real pulses in the segment number 1 (say,) are used as the triggers in the oscilloscope.

Earlier authors have discussed [4, 5], that double peaking is observed when addback spectra are reconstructed from the two-fold events in a composite planar detector. However, in their work [ 他, the second peak originated from cross-talk between two neighbouring segments. Cross talk induced second peak usually appears at higher energy compared to the original one, due to its positive nature. However, in the present case, the second peak is at lower energies (Fig. 3), indicating effect of image charge formation.

\subsubsection{Image charge formation}

For segmented detectors, along with a net charge signal from the collecting electrode, one also gets transient signals of neighboring segments where image charges are induced. These transient signals are utilised to get position information of the interaction point of the gamma in the detector. The transient pulses corresponding to image charge formation, are of opposite polarity to the original pulses. The formation of the pulses corresponding to image charges is evident when the pulses in 
two segments of the LEPS detector are observed simultaneously if one is triggering the other, in the oscilloscope. Such characteristic of a segmented planar detector has also been discussed by R.J. Cooper et al.. [4. 5]. For Compton scattered events which are detected in two neighbouring crystals, their sum peak may have peak height lower than the actual gamma energy due to the presence of simultaneous negative pulses originated from image charges. The variation of the heights of the image pulses with change in the initial interaction position of the gamma in the detector has been important in developing imaging and or tracking detectors [5].

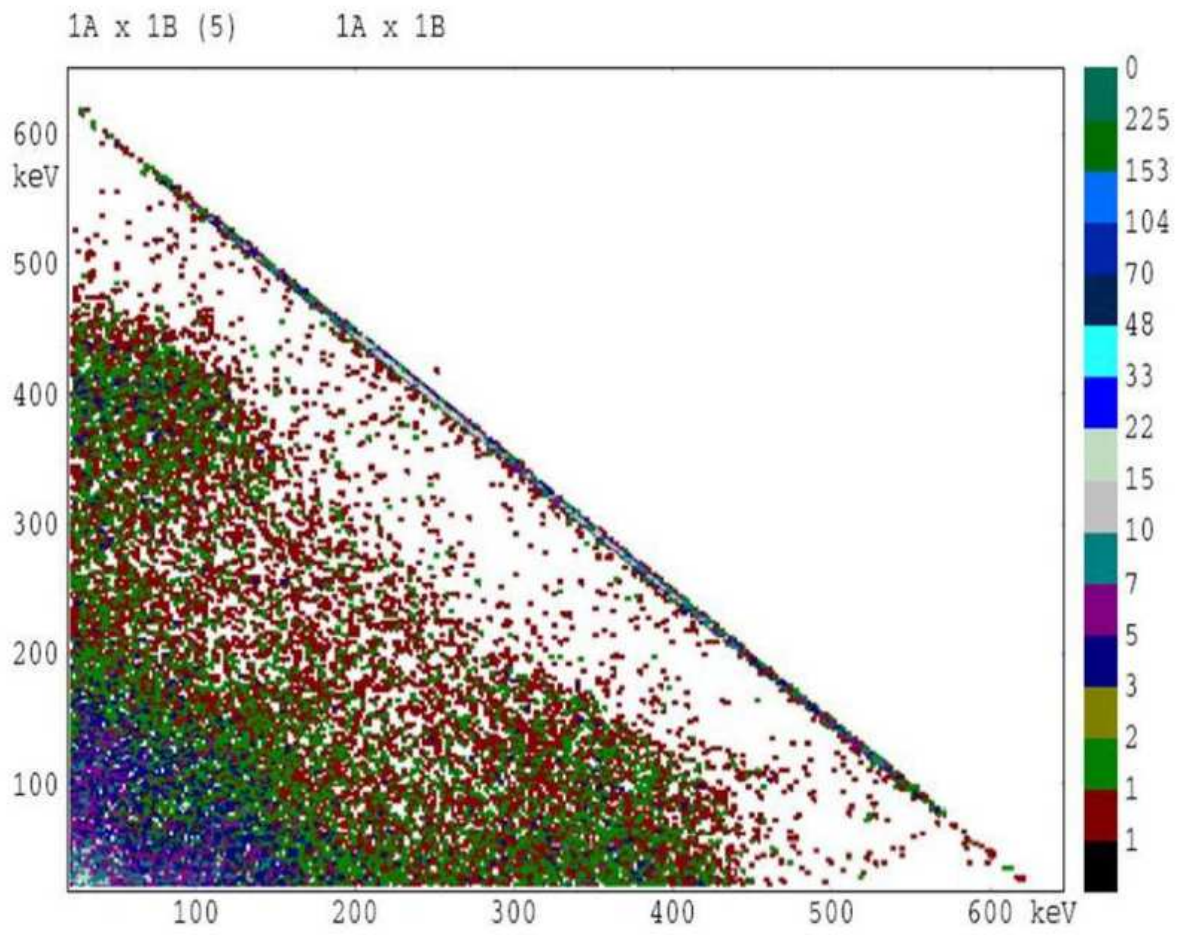

Figure 6. A gamma- gamma two dimensional spectrum showing the simultaneous events recorded by two crystals of the detector for $662 \mathrm{keV}$ gamma from a ${ }^{137} \mathrm{Cs}$ source.

A two dimensional matrix have been generated from the correlated events with a ${ }^{137} \mathrm{Cs}$ source, and is plotted in Fig.6. It shows an intense diagonal line running from the top left to the bottom right corresponding to true Compton scattered correlated gamma events detected in two adjacent segments which add up to a total energy of $662 \mathrm{keV}$.

\subsubsection{Correction}

The magnitude of the shift of the shadow peak originated due to image charges was calculated by plotting the energy of the second peak (in $\mathrm{keV}$ ) as a function of gamma ray energy. This result is displayed in Fig. 7, showing the proportionality nature of the shift. The gradient of the fitted line indicates that the magnitude of this shift is only $\simeq 2 \%$ of the deposited energy.

This energy shift in the peak generated from the two fold events can be compensated by applying an event by event correction in the calculated addback energy. The effect of this correction can 
be seen in Fig. 8. It is seen that the correction removes the events from the shadow peak to improve the count of the full energy peak. The full energy peak for $356 \mathrm{keV}$ gamma from a ${ }^{133} \mathrm{Ba}$ source has been compared with the same photopeak after reconstruction including the corrected two - fold events (Fig. 8). However, at higher energies, gradually the base of the peak widens and the FWTM (full width at one-tenth of maximum) deteriorates after correction. This is due to the shift variation according to the relative location of the two segments of the detectors fired simultaneously. Thus, the corrections need to be different depending on this relative position. In the present case, shown in Fig. 8, different pairs were not distinguished and the same correction factor was used for any two -fold event depending on the incident energy. The variation of the heights of the image pulses with change in the initial interaction position of the gamma needs to be considered for better reconstruction of the spectrum.

\subsubsection{Addback Factor}

This correction factor improved the performance of the composite LEPS detector which has been estimated by the addback factor as usually defined in composite detectors [设]. An estimation of the variation of the addback factor with increase in incident gamma energy is shown in Fig. 9 .

\section{Conclusion}

In the present study, the composite LEPS has been characterised using radioactive sources up to 1.4 $\mathrm{MeV}$. Formation of image charges in neighbouring segments deteriorates the performance of the detector. Event-by-event correction has been implemented. The corrected results indicate that these detectors can also be used in addback mode with an increase in efficiency by $\simeq 20 \%$ at $1.408 \mathrm{MeV}$.

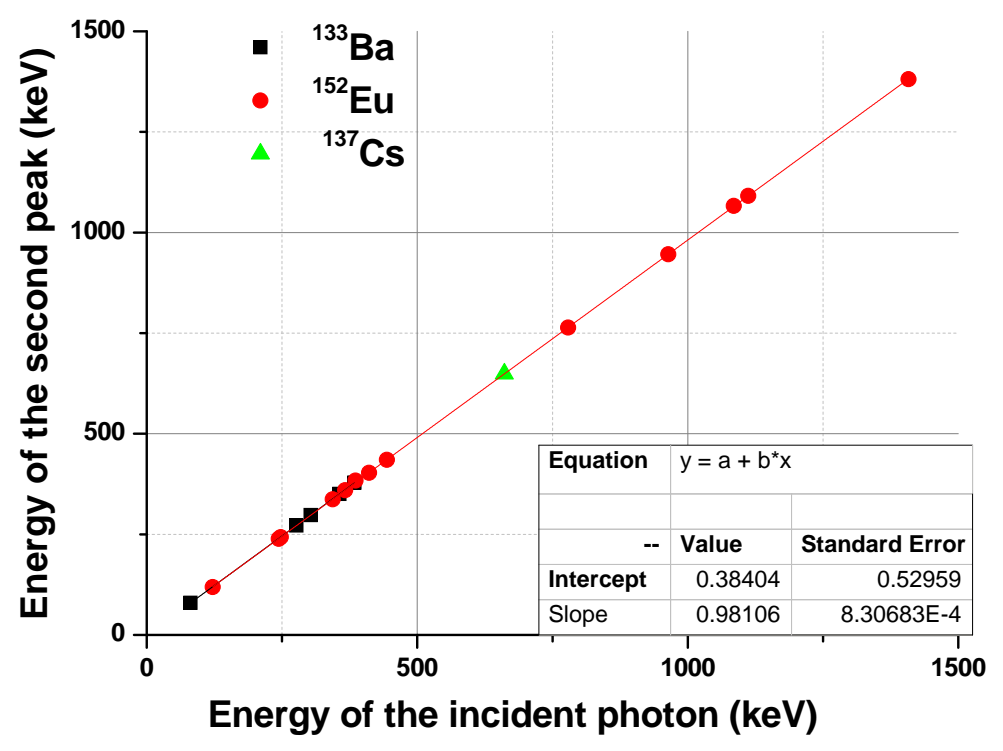

Figure 7. Plot showing the energy of the second shifted (shadow) peak as a function of energy of the original peak. 


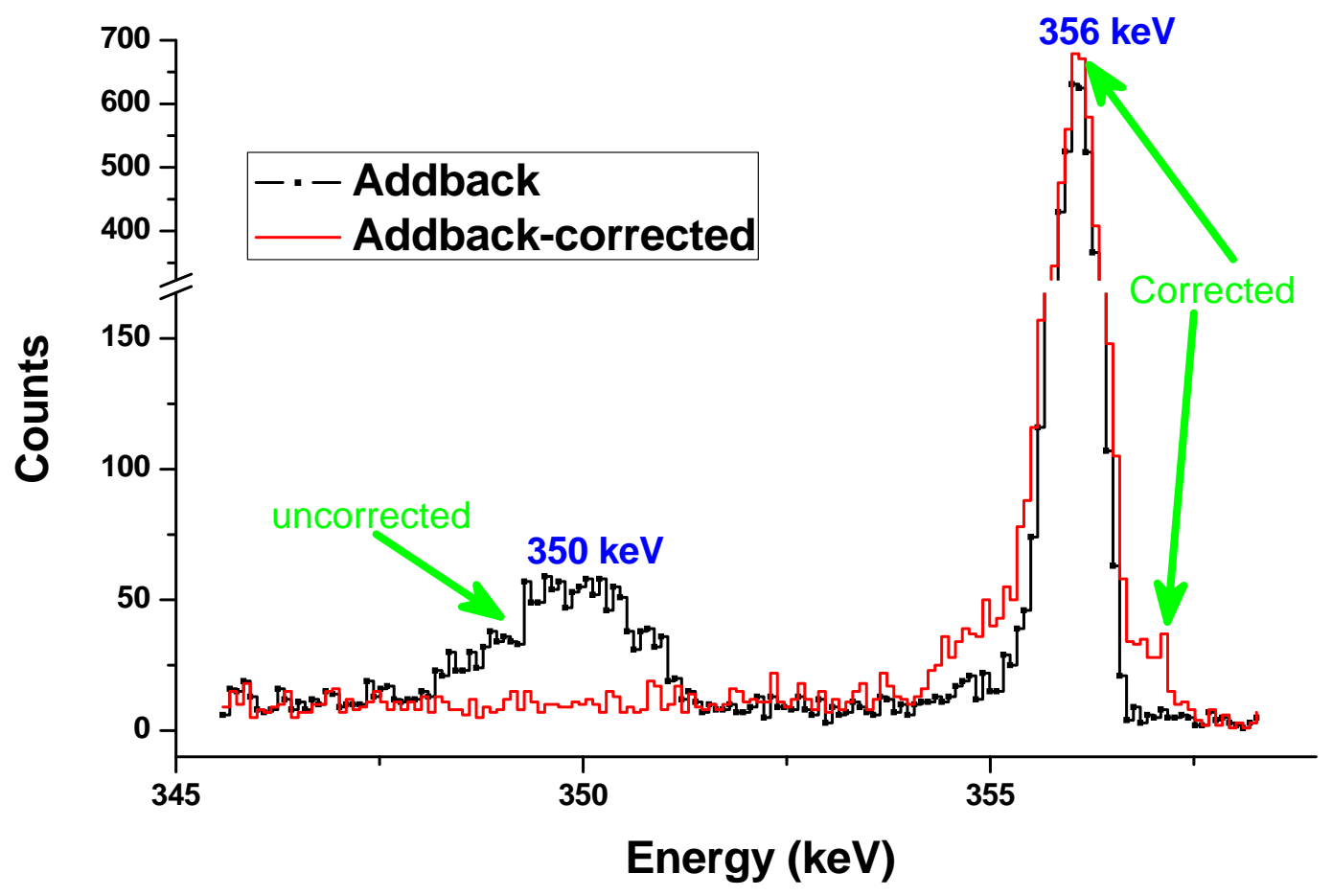

Figure 8. Spectra showing the $356 \mathrm{keV}$ photopeak from ${ }^{133} \mathrm{Ba}$ in the addback spectra before (contains a second shadow peak at lower energies) and after shift correction.

However, to improve the peak shape of the reconstructed peak, one may use different correction factors for different relative locations of the two segments of the detectors fired simultaneously.

\section{Acknowledgments}

The authors sincerely thank Ms. Jonaki Panja for technical help during the experiment.

\section{References}

[1] http://www.tlabs.ac.za/nuclear-physics-afrodite/; Muralithar S et al., Indian National Gamma Array at Inter University Accelerator Centre, New Delhi 2010 Nucl. Instr. and Meth. A 622281.

[2] Saha Sarkar Met al., Characteristics of a Compton suppressed Clover detector up to $5 \mathrm{MeV} 2002$ Nucl. Instr. and Meth. A 491113 ; Characterisation of a Compton suppressed Clover detector for high energy gamma rays $\leq 11 \mathrm{MeV}, 2006$ ibid., 556266 and references therein.

[3] Saha Sarkar M, Ray Sudatta, Bhattacharya Purba, Roy Basu Moumita, Bisoi Abhijit, Characterisation of a Novel Composite LEPS, 2009 Proc. DAE-BRNS Int. Symp. Nucl. Phys. (India) 54 656; http://www.sympnp.org/proceedings/54/H9.pdf

[4] Cooper R J et al., Charge collection performance of a segmented planar high-purity germanium detector, 2008 Nucl. Instr. and Meth. A 595401 and references therein. 


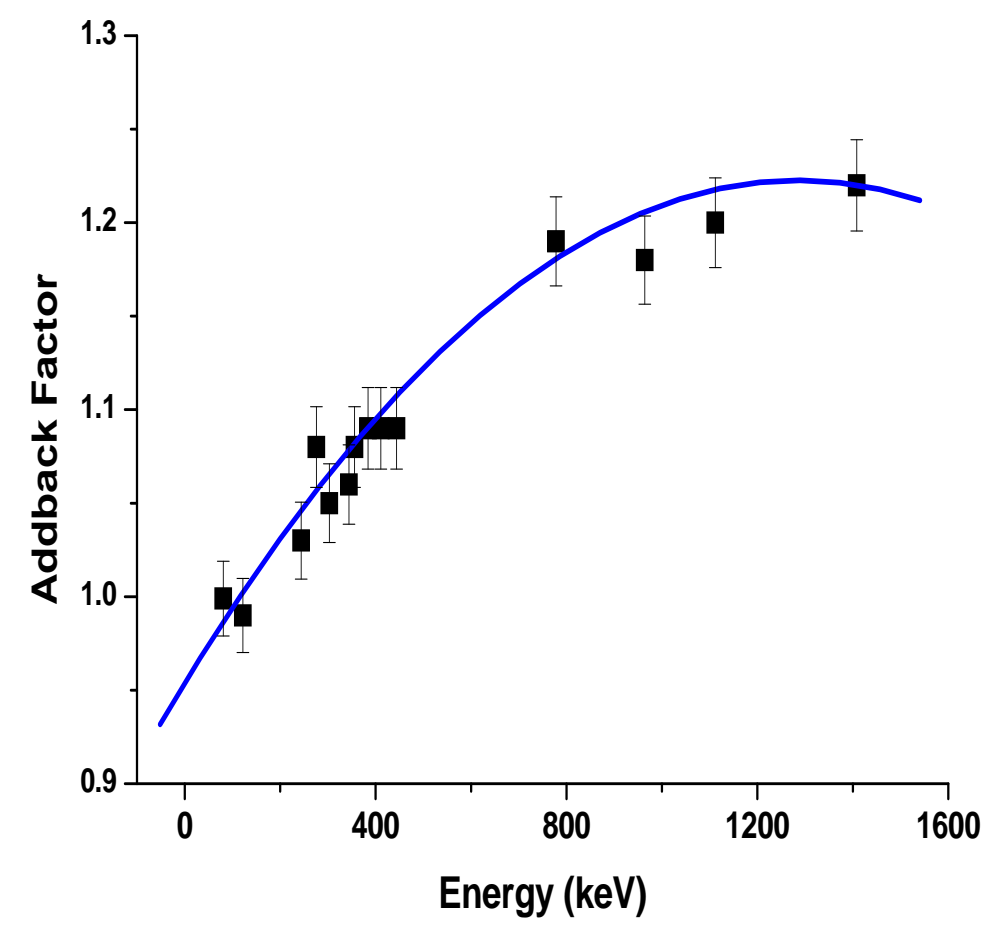

Figure 9. Variation of the addback factor as a function of energy, after shift correction.

[5] Cooper R J et al., SmartPET: Applying HPGe and pulse shape analysis to small-animal PET, 2007 Nucl. Instr. and Meth. A $\mathbf{5 7 9} 313$. 